\title{
Lumen
}

Selected Proceedings from the Canadian Society for Eighteenth-Century Studies

\section{Idéologies et sociétés au Québec au XVIII ${ }^{\mathbf{e}}$ siècle}

\section{Fernand Ouellet}

Volume 15, 1996

URI : https://id.erudit.org/iderudit/1012480ar

DOI : https://doi.org/10.7202/1012480ar

Aller au sommaire du numéro

Éditeur(s)

Canadian Society for Eighteenth-Century Studies / Société canadienne d'étude du dix-huitième siècle

ISSN

1209-3696 (imprimé)

1927-8284 (numérique)

Découvrir la revue

Citer cet article

Ouellet, F. (1996). Idéologies et sociétés au Québec au XVIII ${ }^{\mathrm{e}}$ siècle. Lumen, 15, 161-183. https://doi.org/10.7202/1012480ar d'utilisation que vous pouvez consulter en ligne.

https://apropos.erudit.org/fr/usagers/politique-dutilisation/ 


\section{Idéologies et sociétés au Québec au XVIIIe siècle}

Au Québec, comme en France et aux Etats-Unis, le XVIIIe siècle eut une place de choix dans l'historiographie. Ici, ce fut moins par le volume des publications consacrées à ce siècle que par la signification attachée à un événement dramatique, la conquête anglaise de 1760, que cette transcendance fut acquise. En effet, beaucoup plus que les révolutions française et américaine, cet événement fut au coeur des reconstructions du passé faites de 1830 jusqu'au moment de l'entrée ferme, vers 1980, de la société québécoise dans la modernité. Ici en plus, l'image projetée de ces révolutions par les classes dirigeantes et les intellectuels francophones, loin d'avoir été jusqu'à la révolution tranquille de 1960 inspiratrice de renouveau idéologique et social, fut la plupart du temps largement repoussante. Naturellement, les historiens et les intellectuels anglophones furent davantage préoccupés par le processus d'édification d'une nation canadienne sous l'angle de la diversité culturelle. C'est pourquoi, en relisant le passé, ils accordèrent toujours plus d'importance à la Révolution américaine qu'à la conquête de 1760. N'empêche qu'ils furent loin de respirer à pleins poumons le souffle glorieux de l'une ou l'autre de ces grandes révolutions.

Ainsi, depuis 1840 du côté francophone, des visions successives de ce XVIIIle siècle québécois, presque toujours polarisées autour de 1760, ont été dégagées pour répondre à des besoins présents, sinon de la société dans son ensemble, du moins de certains groupes en quête d'un pouvoir ou, plus simplement, avides de défendre leurs acquis. Ces schémas interprétatifs tenaient, pour une bonne part, du discours idéologique et, comme tels, ils étaient à la fois représentations partielles du passé et porteurs de visées sociales et politiques. Notre objectif n'est donc pas de tout remettre en perspective mais de le faire suffisamment de façon à mieux identifier des acquis et des orientations actuelles de la recherche. 


\section{Race et survivance nationale, 1830-1950}

Dès son premier essai, François-Xavier Garneau émit une interprétation du passé qui, pour l'essentiel, donna le ton à l'historiographie québécoise pendant plus d'un siècle après 1840 . Si cette version lui survécut ainsi, c'est qu'elle répondait à des questions qui restèrent actuelles même quand le Québec francophone fut engagé d'une façon irréversible dans le processus urbain et industriel. ${ }^{2}$

Ainsi, après le début du XIXe siècle, à mesure que se dessina le mouvement nationaliste canadien-français, politiciens et intellectuels éprouvèrent le besoin d'une perspective historique. L'histoire $d u$ Canada du notaire François-Xavier Garneau, parue entre 1845 et 1852 et rééditée à plusieurs reprises, fut autant le reflet de cet univers idéologique que le résultat d'un retour aux sources. On a souvent prétendu que Garneau avait été la conscience historique des patriotes de 1837-1838. Pourtant, presque tout ce qu'il dit à leur sujet est une condamnation. Non seulement les accuse-t-il d'avoir pris le risque le plus grave, celui de l'annexion aux Etats-Unis, mais il les rend partiellement responsables de l'Union des Canadas. ${ }^{3}$ Comme Bédard et La Fontaine, Garneau était un antirépublicain essentiellement préoccupé par la survivance des Canadiens français dans le cadre d'institutions parlementaires britanniques réformées.

Pour Garneau, la suprématie des droits collectifs allait de soi. C'est pourquoi, il portait en lui l'image d'un petit peuple d'une vigueur unique, abandonné par la mère-patrie, sans cesse menacé de l'intérieur et de l'extérieur et, par conséquent, condamné à la survivance. Car, pour lui, la nationalité était un don de Dieu, créateur des races et de leurs attributs les plus chers: la langue, les lois et la religion. ${ }^{4}$

$\mathrm{Vu}$ dans cette perspective, le XVIIe siècle fut le moment où les colons, issus de la première nation $\mathrm{d}^{\prime}$ Europe et des régions où la race française était à son mieux, avaient confectionné leur identité canadienne au gré de leurs luttes. Donc, vers 1700, selon Garneau, ces colons, choisis avec soin, au passé génétique exceptionnel, avaient mis en place une société forte et stable à 'l'image de la France,' dotée 'd'une brave noblesse et $d^{\prime}$ 'une population vraiment nationale, catholique, française, sans mélanges de races. ${ }^{5}$

La première moitié du XVIIIe siècle fut, par conséquent, un moment unique pour ce peuple prolifique de 'laboureurs, de chasseurs et de soldats.' En guerre, sur les pas de leurs seigneurs, ou en marche vers l'ouest pour les fourrures, les Canadiens menèrent une existence plus chevaleresque que celle de leurs voisins anglais. Leur société était, dit Garneau, militaire. ${ }^{6}$ 
A cette époque, les querelles internes sur la position respective de l'Eglise et de l'Etat dans la hiérarchie étaient à peu près vidées. Aussi Garneau, en bon gallican, était-il fier de ce catholicisme national exempt de toute hérésie et fidèle à sa mission originelle: la conversion des 'barbares.' Le clergé était à sa place et, sous l'égide de l'Etat, il remplissait admirablement sa mission dans le ministère paroissial, dans l'éducation, le soin des malades et l'aide aux miséreux. ${ }^{7}$

La seigneurie n'avait pas non plus échappé à ce processus d'embellissement, puisqu'elle avait perdu son caractère féodal, le seigneur n'étant guère plus qu'un 'fidei-commissaire.' Il est vrai qu'après la conquête, les censitaires furent livrés 'à la cupidité des seigneurs.' Mais, le poste d'intendant avait été aboli et la justice n'était plus 'en général administrée d'une manière impartiale et éclairée et surtout à bon marché' comme autrefois, dit Garneau. ${ }^{8}$

Près comme il l'était, des luttes sur la vocation nationale de l'assemblée législative, Garneau n'aurait pu faire, à l'exemple de ses successeurs, l'éloge de l'absolutisme monarchique: le plus mauvais système de gouvernement, disait-il. ${ }^{9} \mathrm{Au}$ total, à part quelques problèmes sérieux, la société de la Nouvelle-France était d'une grande perfection. Ce peuple que la 'Providence ${ }^{\prime 10}$ avait même protégé à un moment tragique, fut donc heureux jusqu'à son abandon par une monarchie décadente.

Pour Garneau, il était inévitable que l'administration et le commerce passent aux mains des Anglais en 1760. Mais ceux-ci, afin de mieux les dominer et exploiter, $\mathrm{s}^{\prime}$ empressèrent de comploter contre la langue et les institutions des Canadiens. C'est à ce moment que débute, dit Garneau, la véritable lutte pour la survivance nationale. Pour résister à cette menace, les Canadiens firent appel à toutes les ressources connues et secrètes de la race. ${ }^{11}$ Grâce en plus au tact admirable de leurs élites, à l'appui des gouverneurs et d'esprits élevés en Angleterre, ils furent capables de surmonter ce péril et se virent octroyer en 1774 l'acte de Québec qui reconnaissait leurs droits.

Mais, pendant ce temps, les colons américains avaient levé l'étendard de la révolte et, pour attirer les Canadiens dans leur camp, ils leur promirent à la fois l'indépendance, la république, la liberté de conscience et le nivellement social. Naturellement, le clergé et la noblesse prirent peur. Ainsi coincés entre les envahisseurs, leurs élites et les dirigeants britanniques, les habitants, qui considéraient tous les Anglais comme 'une même race d'oppresseurs turbulents et ambitieux,' choisirent la neutralité. En vérité, raconte Garneau, les 'Canadiens ne méprisaient pas cette liberté... [Mais une] liberté qui doit anéantir votre nationalité est plus triste qu'un régime monarchique qui peut la laisser subsister. ${ }^{\prime 12}$

Une fois la paix revenue, la lutte pour la survivance prit une allure différente. Car, les libéraux anglophones et francophones joignirent 
leurs efforts pour obtenir des institutions parlementaires. Cette fois encore, nobles et clercs, toujours par intérêt, s'opposèrent à tout changement. Mais ce fut peine perdue et les Canadiens eurent désormais un instrument incomparable pour protéger leur nationalité. ${ }^{13}$

Evidemment, Garneau reconnut pleinement le danger que représentait la révolution française en Europe. Mais il pensait aussi que les Canadiens, enfin en paix et libres, n'avaient pas lieu d'être inquiets. Aussi se montra-t-il particulièrement critique à l'égard des officiels britanniques et des classes dirigeantes coloniales, réagissant de manière excessive au moindre signe d'agitation et d'infraction idéologique.

Cette reconstruction du passé autour du collectif racial et de la conquête de 1760 avait neutralisé le message des révolutions sur les libertés individuelles, l'idée de progrès et une vision libérale de la nation. Et cela, pour faire passer un autre message taillé à la mesure des Canadiens français: être prudent, éveillé au danger, éviter les nouveautés, rester soi-même et ne changer que graduellement. ${ }^{14}$

A l'époque où Garneau écrivait, plus de $85 \%$ de la population habitait en dehors des trois villes bas-canadiennes et, dans celles-ci, la présence francophone dépassait à peine la moitié des effectifs. En 1850, la tendance se renversait et, désormais, la proportion des urbains ne cessa plus d'augmenter. Les Francophones allèrent dans la même direction mais plus lentement que les autres Québécois. Aussi, restèrent-ils davantage concentrés dans les endroits où la densité urbaine et manufacturière était plus faible, étant aussi fortement sous-représentés parmi les chefs d'entreprises comme ils l'avaient été à l'époque française. ${ }^{15}$

On comprend que cette situation ait favorisé la montée, parmi eux, du pouvoir clérical et d'une vision ultramontaine du monde traduite dans les rapports entre l'Eglise et l'Etat. L'hostilité à l'égard de la société urbaine et industrielle, partagée par Garneau, acquit plus d'intensité chez ses successeurs. D'autant plus que les historiens se recrutèrent davantage parmi les clercs et des laïcs qui pensaient comme eux. ${ }^{16}$

Ainsi, d'une décennie à l'autre, l'idée de nation fondée sur la race, la langue et la religion imprégna encore plus le discours sur le passé. Au traumatisme de la conquête, s'ajouta celui engendré par la révolution urbaine et industrielle. Il n'y a aucun doute que l'homme qui incarna le mieux cette réaction, fut l'abbé Lionel Groulx qui qualifia cette révolution pacifique de 'seconde conquête plus désastreuse peut-être que la première. $^{17}$

Ainsi, quand il entreprit sa remontée dans le temps afin d'y sous-peser les chances de survie des Canadiens français à ces chocs répétés, l'un anglais et l'autre américain, son bagage intellectuel incluait les oeuvres de Gobineau et de Maurras. ${ }^{18}$ Arrivé au passé le plus lointain, il y retrouva Garneau. Car, pour lui aussi, les premiers colons étaient issus 
d'une race supérieure, spirituelle, à l'époque où la monarchie française était à son apogée, et ils avaient fait l'objet d'une sélection serrée à tous égards. Ainsi prédisposés, ils n'eurent pas de peine, à travers leurs luttes héroïques, à se donner une forte personnalité canadienne. A cet égard, Groulx disait: 'Du mélange de tous les sangs des meilleures provinces françaises, $l^{\prime}$ on peut déjà prévoir la vitalité puissante de la jeune race canadienne $^{19}$ qui, disait-il, avait par la suite préservé sa pureté originelle: seulement quelques unions avec des aborigènes qui, presque naturellement, avaient porté peu de fruits. ${ }^{20}$

$C^{\prime}$ est de cette façon que s'était dessinée une grande aventure missionnaire et exploratrice à l'échelle du continent. L'épuration des institutions avait aussi contribué à la vigueur de la race. Un catholicisme, de nature presque biologique, retrempé aux sources les plus anciennes et intimement associé à la paroisse et à la famille, un absolutisme tempéré par le paternalisme et un régime seigneurial sans exploitation et dépendance, voilà qui offrait en plus d'incomparables garanties de survie. ${ }^{21}$

Une société d'ancien régime hiérarchisée mais ouverte avait donc pris forme. Aussi la noblesse y jouait-elle un rôle plutôt décoratif. La bourgeoisie était également modeste et le clergé y exerçait avec dévouement et sans jamais abuser, un rôle dominant. Naturellement, l'agriculture était la base de l'économie et la paysannerie, la meilleure au monde, y constituaient le coeur de la nation. Une seule ombre au tableau, presque: le trafic des pelleteries avec les indigènes qui risquait de devenir 'le poison de la race.' La Nouvelle-France de Groulx était donc une théocratie érigée sur un consensus. ${ }^{22}$

La conquête de 1760 marque ainsi la fin d'une grande aventure et cela, principalement, par la faute de la France et de ses agents. Pour Groulx, la question en était désormais une de 'cohabitation des races,' mais sans mélanges. La lutte était, par conséquent inévitable, entre ce jeune peuple 'catholique et français' et des vainqueurs avides de gains matériels et d'imposer leur culture. Comme chez Garneau, elle fut déclenchée en 1763 en réponse à une politique d'assimilation conçue au Canada et adoptée par le gouvernement anglais.

Heureusement, raconte Groulx, ce projet infâme fut abandonné et remplacé par l'Acte de Québec de 1774 pour des raisons similaires à celles invoquées par Garneau. Il est vrai que Groulx attribua un rôle à la Providence dans cette défaite mais, en fin d'analyse, il était d'accord avec lui pour dire que ce fut 'l'énergique réaction du conquis [qui] a ouvert les yeux de l'assimilateur,' le forçant à reconnaître ses droits collectifs fondamentaux. ${ }^{23}$

Sa vision de la Révolution américaine est moins nette que celle de Garneau, parce qu'il ne semble pas se préoccuper autant que lui du péril culturel, auquel elle exposait les Canadiens français. A vrai dire, Groulx 
en était fort conscient mais il voulait, de cette façon surtout, livrer le message que, vu sous l'angle de la liberté politique, l'Acte de Québec avait laissé les milieux populaires sur leur faim. De là son insistance sur le fait que: 'le peuple... sympathisa ouvertement avec l'insurrection.' Un peu comme si celui-ci était déjà, silencieusement, engagé dans la lutte pour les institutions parlementaires à l'encontre des nobles et du clergé. ${ }^{24}$

Pour Groulx, le concept de liberté politique ne référait pas aux droits du citoyen. Car, elle était avant tout incarnée dans l'assemblée législative, porte-parole d'une majorité nationale, qui s'en servait pour obtenir la plénitude de ses droits collectifs. ${ }^{25}$ On comprend qu'il ait été embarrassé puisque, jusque-là, le mouvement qui réclamait des institutions parlementaires, avait été dirigé par la minorité anglophone, auquelle s'étaient ralliés des notables de langue française en nombre croissant. Par contre, le clergé et la noblesse, gardiens de la nationalité, se sentaient menacés par cette réforme et $\mathrm{s}^{\prime} \mathrm{y}$ opposaient. ${ }^{26}$ Mais, grâce à l'immigration loyaliste, aux leçons tirées des deux révolutions et à un fond de scène populaire indéfinissable, les réformistes eurent raison des hésitations de Londres. Malheureusement, disait Groulx, ce premier parlementarisme, 'nouvelle étape [vers] l'émancipation,' était truqué et, peu à peu, se dessina la longue lutte qui devait mener au gouvernement responsable. ${ }^{27}$

Cet effort de prise en main politique s'imposait d'autant plus que, d'après Groulx, la conquête avait infléchi l'évolution des Canadiens français dans plusieurs domaines essentiels de la vie collective. Aussi fait-il état des mariages mixtes, du déclin des moeurs, de l'éducation, de l'influence des philosophes et des idées révolutionnaires. ${ }^{28}$ A propos de ceux qui avaient bu à la santé de la Révolution française, sa réprobation fut totale: 'Quelles naïvetés et combien lourdes de désenchantements! Ces esprits livresques ont cru à la liberté telle qu'entrevue dans les utopies des Encyclopédistes.... ${ }^{29}$

Dans la même tradition, mais se tenant plus près de Garneau que de Groulx, le livre de Guy Frégault La civilisation de la Nouvelle-France paru en 1944, reprend, en les intégrant, l'essentiel de leurs visions respectives. Dans son schéma figuraient l'esprit de la race, l'épuration des institutions, une société coloniale ouverte, militaire, féodale sans ferments d'oppression et d'exploitation, un absolutisme tempéré, un clergé puissant, mais moins que la classe seigneuriale, et une bourgeoisie minuscule. 'Seigneurs et censitaires forment l'élément de base de l'organisation sociale du pays,' disait celui qui deviendra un des piliers de l'Ecole néo-nationaliste. ${ }^{30}$ 


\section{Survivre n'est pas vivre: les néo-nationalistes,1950-1970}

Cette tradition de la survivance, qui ne devait rien au message de 1789 sur la nation, fut remise en question vers 1950. Car, à cette date, l'attention de la nouvelle petite bourgeoisie et des intellectuels était polarisée par le sentiment des retards du Québec francophone et de la faiblesse de sa bourgeoisie industrielle. Pour la plupart, cette prise de conscience appelait le procès des institutions traditionnelles et de profondes réformes internes. Pour d'autres, au contraire, elle incitait à rêver d'indépendance et de l'avènement d'une classe bourgeoise dominante, maître de $l^{\prime}$ Etat et créatrice de modernité. 'Survivre n'est pas vivre,' déclarait Michel Brunet. ${ }^{31}$

Les fondateurs montréalais de $\mathrm{l}^{\prime} E$ Ecole historique néo-nationaliste, avaient eux aussi repris la route du passé avec quelques certitudes en tête. Notamment, que la nation avait ses racines 'dans la nature de l'homme' et que la bourgeoisie capitaliste avait été la 'classe dirigeante du monde occidental moderne.' A vrai dire, leur idée de nation, justifiant la voie indépendantiste, ne se démarquait pas tellement de celle de Groulx, puisque le mot 'nature' y avait simplement remplacé le mot 'race' et que la bourgeoisie, non le clergé ou la noblesse, était dans ce monde idéal la classe dominante. ${ }^{32}$

Ainsi, pour ces historiens, la Nouvelle-France était une création de la France moderne et bourgeoise. Toutes deux étaient si rapprochées par les liens du sang que la mère patrie n'aurait pu se livrer à l'exploitation des colons. A l'intérieur de la colonie, le même esprit d'harmonie et de générosité animait les rapports sociaux. La bourgeoisie y régnait mais, associée à un Etat plein de vigueur, elle devait plutôt son ascendance à la mise en valeur des ressources du territoire, particulièrement les pelleteries. C'est dans ce climat de mutualité que s'était formée la nation canadienne, un peu comme si la métropole avait prévu et préparé son éventuel accès à l'indépendance. Il n'y eut donc pas, en 1760, abandon de la colonie par la France, puisqu'elle avait simplement succombé sous le poids du nombre. ${ }^{33}$

Quitter cet univers naturel, chaleureux et moderne pour se retrouver subitement en 1760 sous le joug étranger, privée de sa classe dominante, constituait, à n'en pas douter, un traumatisme si violent pour cette jeune nation qu'elle en fut brisée et vouée à une existence presqu'anémique jusqu'au jour lointain de l'indépendance réparatrice. En effet, disait Brunet, les Britanniques avaient conquis le Québec avec l'intention de le coloniser, de le développer à leur profit et de modeler les conquis à leur image. Grâce à leur contrôle du pouvoir politique, ils mirent la main sur l'économie, en exclurent les marchands canadiens de son contrôle et mirent le clergé en tutelle. Leur plan aurait pu fonctionner s'il y avait eu 
une forte immigration britannique. Mais, à défaut de celle-ci et ayant à faire face à l'agitation américaine, les dirigeants britanniques furent obligés de composer avec les vaincus. L'Acte de Québec était le résultat de ces circonstances, de la résistance passive du peuple et de l'opposition imprévue des élites canadiennes aux mesures qui affectaient leurs intérêts. Ceci dit, les Canadiens, bien que bénéficiaires du pacte aristocratique, s'en retrouvèrent quand même à la suite de ces événements divisés et coincés entre l'absolutisme des gouverneurs qui les soutenaient et les pressions de la minorité assimilatrice. C'est l'époque où, dit-il, le pouvoir clérical prit de l'ampleur. ${ }^{34}$

Ce type de nationalisme se retrouve chez Pierre Tousignant, mais entremêlé avec une vision plus libérale de la nation et de la société. Ainsi, selon lui, dès la fin de la révolution américaine, les réformistes canadiens s'étaient associés, mais à leur propre compte, au mouvement en faveur des institutions parlementaires. Leur idéologie était, dit-il, à la fois nationaliste et libérale, contrastant ainsi avec celle du clergé et de la noblesse. Leurs sympathies pour la philosophie des Lumières et l'oeuvre de 1789 étaient réelles mais elles se portèrent finalement sur la notion de liberté véhiculée par la constitution britannique. ${ }^{35}$

Le discours de Jean-Pierre Wallot va plus loin, mais dans le même sens. Car, lui aussi pensait que l'après-conquête avait déclenché une lutte de nation contre nation à l'intérieur d'une 'colonie-garnison.' Malgré ou pour cela, les Canadiens avaient été sensibles au message des Lumières et des deux grandes révolutions du siècle. Aussi Wallot décrit-il, le sentiment populaire des années de la révolution américaine comme pré-révolutionnaire. Mais, ajoute-t-il, le déclin de la bourgeoisie commerciale canadienne, l'opposition des clercs et des seigneurs et, surtout, 'l'absence de leaders' contribuèrent à désamorcer cette fièvre. A tel point que la réforme de 1791 sera faite sous le simple signe de la 'liberté britannique.' Il est vrai que les Canadiens français, qui avaient pris part à cette réforme, célébrèrent avec brio les premiers instants de la révolution française. Mais, lorsque celle-ci devint républicaine et que les paysans canadiens s'agitèrent occasionnellement en des gestes qualifiés de révolutionnaires, 'personne n'était là, dit-il, pour les conduire.' Tout cela est d'autant plus inexplicable que, selon Wallot, les notables nationalistes et libéraux canadiens-français d'avant 1783 et d'après 1789 avaient des vues plutôt radicales et qu'un des partis politiques existant aurait pu être utilisé pour canaliser ces sentiments. Il est évident que Wallot exagère beaucoup, notamment en ce qui concerne l'existence d'authentiques partis politiques à cette époque et la représentativité de l'idéologie de Mesplet et Jautard, immigrants français, qui dirigeaient la Gazette de Montréal. Notons aussi que cette bourgeoisie, soit-disant décapitée en 1760, affiche, si on suit Tousignant et Wallot, une maturité 
et une vigueur idéologique surprenantes. ${ }^{36}$ Un peu mieux étoffé à certains égards, le livre de Murray Greenwood, Legacies of fear, rédigé dans la foulée des écrits de Wallot, convie aux mêmes interrogations.

\section{Une vision économique et nationaliste: Donald Creighton}

Ce XVIIIe siècle présenté par l'historiographie nationaliste francophone d'avant 1970 contraste à bien des égards avec l'image qu'en avait proposée Donald Creighton peu après 1930. Car, sa vision, inspirée de la théorie des 'staples,' en tant qu'instrument de conquête de la bourgeoisie, était d'abord polarisée autour de la minorité anglophone et des forces économiques et géographiques qui avaient suscité le développement d'une nation canadienne composée de groupes ethniques différents. ${ }^{38}$

En effet, c'est grâce à un produit de base commercialisé dans la métropole, en l'occurrence les pelleteries, et à l'orientation du réseau hydrographique qu'une économie transcontinentale avait pu naître et s'étendre. La société coloniale était, par conséquent, à la fois une réplique de celle de la mère-patrie et originale. Comme les pelleteries étaient la source principale d'accumulation du capital et l'agriculture seulement une activité de subsistance, les traditions féodales n'avaient pu que se détériorer peu à peu. Il va de soi que la bourgeoisie avait pris le pas sur la noblesse et le clergé. Creighton s'accordait aussi avec les nationalistes québécois pour dire qu'en 1745 une nationalité s'était constituée dans la vallée du Saint-Laurent.

Cette conception de la société de la Nouvelle-France s'accordait avec celle des néo-nationalistes non seulement sur le rôle de la bourgeoisie mais aussi sur le fait de la décapitation sociale. C'est pourquoi, Creighton avait affirmé qu'après 1760 seuls des débris féodaux étaient restés de la société antérieure. Mais, pour lui, ce n'était qu'un moindre mal puisque les structures de l'empire commercial du Saint-Laurent avaient été renforcées par l'apport d'une métropole plus puissante et d'une nouvelle bourgeoisie plus dynamique.

$\mathrm{Au}$ fond, Creighton était surtout intéressé par les programmes et les stratégies de cette classe dominante, créatrice de nation, dont l'objectif était le développement pour le plus grand profit de tous de l'empire commercial du Saint-Laurent. Ici, la lutte pour la survivance avait été remplacée par l'affirmation d'une classe marchande consciente des intérêts de l'empire commercial du Saint-Laurent contre les officiels anglophones qui, disait-il, défendaient un ordre politique et social autoritaire, militaire, rural et féodal représenté par la noblesse et le clergé catholiques. Ce conflit, reproduit plus tard dans le Haut-Canada, était 
économique et social avant d'être ethnique et il opposait les intérêts agricoles et ruraux aux intérêts commerciaux et urbains.

Pour Creighton, écrivant dans la perspective de la formation d'une nation canadienne, la Révolution américaine était encore plus significative que la conquête de 1760. Car, elle avait été à l'origine d'une restructuration de l'économie coloniale. En plus de déclencher le déclin de la traite par le transfert de postes stratégiques aux Américains, elle avait favorisé non seulement la croissance rapide de l'économie forestière mais, grâce aux Loyalistes, l'émergence d'une agriculture commerciale.

Naturellement, tout en reconnaissant le rôle des marchands canadiens-français dans le mouvement pour les institutions parlementaires, il insiste d'abord sur le leadership de la bourgeoisie britannique. Là encore, celle-ci fut doublement déçue. Elle l'avait été par l'Acte de Québec et par le traité de 1783. Elle le fut tout autant par la division du Québec en deux provinces et par l'utilisation subséquente de l'assemblée législative bascanadienne par la petite bourgeoisie pour protéger l'héritage de l'Ancien Régime.

Ainsi, le thème de Creighton, c'est celui de la bourgeoisie conquérante poursuivant à l'époque française et pour longtemps encore, inconsciemment sans doute au XVIIIle siècle, un rêve national canadien.

\section{Derrière l'écran idéologique}

Ce besoin incessant d'accorder le passé au présent et au futur s'est maintenu jusqu'à aujourd'hui. Dans les années 1970, stimulés par le marxisme, les espoirs d'indépendance furent placés dans la classe ouvrière. Aussi, les historiens du XVIIIe siècle fixèrent-ils, pour un temps, leur attention sur la paysannerie et les artisans. Comme les plus nationalistes d'entre eux faisaient découler l'exploitation sociale de la domination nationale, leur vision de la Nouvelle-France était aussi idéale que celle de Groulx et des néo-nationalistes. Denis Monière et Marcel Rioux représentent bien ce courant qui fait remonter le début de l'exploitation sociale à la conquête. ${ }^{39}$

Depuis 1980 cependant, les idées de modernisation et de culture ont tellement préoccupé les historiens que la conquête fut apparemment reléguée dans l'ombre. A cette date, la modernisation du Québec était si avancée qu'il n'était plus possible de la faire dépendre de l'accès à l'indépendance. Dès lors, le passé devint celui de la marche silencieuse, quoi que semée d'embûches, de la nation vers la modernité et l'indépendance. ${ }^{40}$ A vrai dire, la professionnalisation de la discipline d'après 1945 n'avait pas contribué à chasser les perspectives idéologiques des travaux sur le passé. Le peut-elle? Comment, alors, parvenir à percer ces écrans 
pour arriver à proposer une image un peu moins approximative de ce XVIIIle siècle?

Jusqu'en 1960, les historiens ont exalté la vigueur reproductrice, les bonnes moeurs et l'homogénéité de la race transplantée dans la vallée du Saint-Laurent. Depuis ce temps, les perspectives ont changé sensiblement et les taux de nuptialité, de natalité et de mortalité, calculés autrefois, ont été revus à la baisse. ${ }^{41}$ Les nouveaux taux, encore fort remarquables, sont quand même en ligne avec les conclusions de l'équipe Charbonneau qui a démontré que les pionniers du XVIIe siècle avaient eu une fécondité plus élevée et une mortalité plus basse que celles de leurs comtemporains de France. ${ }^{42} \mathrm{Au}$ XVIIIe siècle, alors que l'immigration compta pour très peu dans la croissance, la population augmenta à un rythme de $2.6 \%$ par an, soit une natalité d'environ 50 pour 1,000 et une mortalité de 25 . Donc, selon le critère du lieu de naissance, une population d'origine française qui se canadianise au XVIIIe siècle. On ne peut en dire autant de la population anglophone qui, venue après 1760 , se recruta surtout par immigration et dont les performances, quant aux composantes de la croissance naturelle, étaient plus modestes que celles des francophones. ${ }^{43}$

Disons aussi que, dès l'origine, cette population francophone s'est peu à peu déplacée pendant près de deux siècles vers les campagnes. Les pionniers, surtout les femmes, étaient plus urbains que les Francais du temps. Mais leurs descendants, attirés qu'ils étaient par la grande abondance des terres, le furent moins qu'eux. Cette marche vers les campagnes fut aussi reprise par la suite par les immigrants de France. En 1665, le taux de ruralité était de $63 \%$, en 1706 de $75 \%$ et en 1800 de $87 \%$ ). ${ }^{44}$ Ainsi, la ruralisation de la société était un phénomène de longue durée et $\mathrm{d}$ 'une énorme portée culturelle. Elle était si peu liée à la conquête de 1760 que les immigrants anglophones refirent par la suite, mais d'une façon moins marquée, la même expérience. En 1850, ils étaient ruraux dans une proportion de $74 \%$ et les Francophones de $88 \%$.

Donc, une population francophone prolifique, qui se ruralise et qui, tout considéré, occupe le terroir seigneurial en se déplaçant progressivement de la région de Québec vers Montréal. En 1667, les deux tiers de la population rurale se trouvaient dans le district de Québec. En 1800, au contraire, plus de la moitié des habitants était concentrée dans la région de Montréal. Trouver des conjoints à l'extérieur des communautés établies, des terres pour les défavorisés du partage successoral et pour les sans-terres chassés par la saturation démographique, expliquent ce processus migratoire. Les communautés rurales nées de cette mobilité devinrent aussi, avec le temps, très structurées et concentrées sur ellesmêmes. Puis, dans un autre temps, elles furent forcées de jeter du lest. ${ }^{45}$ Vue sous cet angle, cette marche vers l'ouest, c'est-à-dire Montréal, fut 
aussi celle de la cohésion et du contrôle social. Le fait qu'avant 1760 plus de la moitié des procès de paternité et les deux tiers des délits criminels connus eurent lieu dans le district de Montréal illustre bien cette réalité. ${ }^{46}$ Encore en 1800, en dépit du chemin parcouru, les traits urbains de Montréal étaient toujours encore moins affirmés que ceux de Québec. Notons aussi que les anglophones se déplacèrent plus spontanément vers cette région que ne l'avaient fait les francophones.

Tous ces mouvements étaient donc inséparables de ceux de l'économie. En ce domaine, bien que l'agriculturisme ait été répandu parmi les historiens, il en fut quelques-uns, tels Harold Innis, Donald Creighton et Michel Brunet, qui prétendirent que le commerce des pelleteries était la base principale de l'économie coloniale. Plus récemment toutefois, l'idée d'une économie dualiste a fait son chemin. ${ }^{47}$ Agriculture et traite apparurent ainsi comme les deux pôles principaux du développement économique et social. Plutôt étroitement cloisonnée à l'origine, cette économie le devint de moins en moins au XVIIIe siècle. ${ }^{48}$

Le Québec d'avant 1800 fut le seul endroit sur le territoire canadien actuel où le blé exerça dès le départ une extraordinaire suprématie sur la production agricole. Partout ailleurs, même dans le Haut-Canada, les colons pratiquèrent une agriculture mixte. Mais, au Québec, que les sols fussent appropriés ou non, le blé occupa en tous lieux de 65 à $75 \%$ de la récolte de grains et de racines. Car, cette denrée était non seulement centrale dans l'alimentation mais elle avait une valeur commerciale, servant en plus à payer en partie les seigneurs, les curés et les achats chez le marchand.

Avec le temps, le blé eut son marché extérieur. Encore modestes jusqu'en 1747, environ $10 \%$ de la récolte selon nos calculs ${ }^{49}$ et interrompues par la suite jusqu'à la fin du régime français, les exportations reprirent leur ascension de 1765 jusqu'au début du siècle. En 1774 et 1801, elles étaient six et douze fois plus élevées qu'en 1734. Cette expansion favorisa la diversification des occupations dans les campagnes et la multiplication des villages au rythme de la croissance démographique. On comprend que les marchands devinrent encore plus intéressés à acquérir des seigneuries et des censives. ${ }^{50}$

Evidemment, l'égalitarisme paysan, tout relatif qu'il ait été au départ, fut loin d'être stimulé par cette évolution. Au contraire, ceux qui avaient le plus de terre et produisaient davantage furent les premiers gagnants. Les autres ou leurs fils, une majorité aux dimensions variables, combinèrent, comme dans le passé, l'agriculture et le travail saisonnier dans la traite, la forêt ou la pêche, afin de se procurer un revenu d'appoint.

Donc, entre l'agriculture centrée sur les basses terres du Saint-Laurent et le commerce des pelleteries avec sa vision transcontinentale du pays, des ponts furent érigés. Car, ce dernier fut toujours la source principale 
d'accumulation du capital, d'emplois saisonniers et de contacts avec les aborigènes. ${ }^{51}$ En 1700 , les exportations de pelleteries se chiffraient à 200,000 peaux, dont la moitié en castor; en 1798, elles étaient quatre fois plus élevées dont un sizième en castor. La main-d'oeuvre saisonnière utilisée dans ce trafic était, par conséquent, nombreuse, à bon marché et elle était à plus de $85 \%$ recrutée dans les campagnes parmi les agriculteurs voués à la subsistance. Avec le temps, cependant, le réservoir d'engagés se contracta sous l'effet de la commercialisation du blé et les salaires s'accrurent en conséquence. D'autant plus que la compétition avec les Américains et la Compagnie de la Baie d'Hudson augmentait en intensité. Pour résoudre ces problèmes, les commerçants de Montréal firent une petite révolution dans les moyens de transport et ils se regroupèrent dans des entreprises toujours plus considérables. Jusqu'au jour où, vers 1785, la Compagnie du Nord-Ouest devint maîtresse de ce commerce.

On pourrait croire que la structure sociale issue de ces éléments divers était fort originale. Pourtant, on avait affaire à une société d'Ancien Régime, avec ses Ordres, avec aussi certains traits plus archaïques que ceux de la métropole. ${ }^{52}$ La noblesse était à sa place. ${ }^{53}$ Sa présence près du pouvoir était d'autant plus normale que les représentants du roi étaient eux-mêmes des aristocrates. ${ }^{54}$ Cette noblesse avait de nombreux antécédents militaires et, stimulée par la conjoncture, elle put se développer dans le sens de ses traditions. Au XVIIe siècle, elle l'avait fait dans le corps des officiers de milice. Mais, bientôt, elle trouva dans les troupes de la marine un cadre plus conforme à ses aspirations. Tout cela sans compter qu'elle reçut tellement de seigneuries, qu'en 1760 elle contrôlait plus de la moitié du territoire seigneurial. Finalement, comme le roi avait assimilé la traite au commerce extérieur, les nobles purent y participer ouvertement ou en prenant avantage de leur position à titre de commandants des postes de l'ouest.

On a souvent oublié que la noblesse était aussi très présente dans le clergé, surtout dans la haute hiérarchie. En effet, le clergé constituait le premier des Ordres, lié à une Eglise gallicane unie à l'Etat. Il avait de droit la charge des paroisses, le monopole sur l'éducation, le soin des malades et la charité publique. Pour ces raisons, les clercs séculiers et réguliers exercèrent leur droit de percevoir la dîme et les honoraires attachés aux fonctions curiales. Ils reçurent en plus, dans les endroits les mieux situés, de nombreuses seigneuries équivalant au quart du territoire seigneurial où ils exerçaient à la fois les fonctions curiales et seigneuriales avec leurs justices. Au début, leur recrutement se fit surtout en France. Mais, vers 1700, la canadianisation du clergé, surtout séculier, était déjà en marche. Pendant la première moitié du siècle, la proportion des clercs nés au pays fut de $40 \%$ en moyenne; par la suite jusqu'à 1810, 
elle doubla. La tendance fut la même pour les religieuses. Par contre, le clergé régulier mâle résista à ce courant et vit sa position décliner. A vrai dire, ce clergé canadien était originaire des villes pour les trois quarts, du district de Québec pour les trois cinquièmes et de la noblesse et la bourgeoisie pour plus de la moitié de ses effectifs. Notons aussi la sur-représentation dans ce groupe des fils d'artisans urbains et la quasiabsence de ceux des paysans parmi ses membres. ${ }^{55}$

Dans cette structure, l'embourgeoisement de la noblesse n'eut pas lieu et la bourgeoisie y s'y maintint au troisième rang. Il est vrai que les marchands eurent tendance à vouloir se fondre dans cette classe. Pour arriver à l'anoblissement officiel, partiel ou même imaginaire, ceux qui croyaient avoir des chances de promotion, mobilisèrent tous leurs crédits: expérience militaire à titre d'officier de milice, réussite dans les affaires, mariages et achats de terres nobles. Mais, en agissant ainsi, loin de transformer la noblesse, ils furent simplement assimilés par elle.

Une telle hiérarchie est impensable sans la présence au sommet d'un Etat monarchique et absolutiste, auquel il existait peu de contrepoids réels. C'est un fait que confirme Louise Dechêne: 'L'hypercentralisation et le durcissement progressif des moyens d'action s'inscrivent dans la logique d'un pouvoir autoritaire que rien n'empêche de s'abandonner à ses penchants naturels. ${ }^{56}$ Pas même l'intendant n'a pu jouer ce rôle modérateur, selon les monographies seigneuriales récentes, qui attribuent aussi à la seigneurie canadienne en voie de mûrissement la même substance qu'à sa contrepartie française. ${ }^{57} \mathrm{Il}$ est vrai que les bras de la justice royale ne s'étendaient guère au-delà des villes. Au civil, les élites urbaines étaient les principales clientes de la cour, alors qu'au criminel ce furent les immigrants et les soldats. Mais, dans les deux cas, les paysans échappaient pratiquement à l'emprise du système. Il est incontestable qu'il y eut une chute substantielle de la criminalité officielle avant et après 1760 . A vrai dire, ce déclin reflétait simplement la ruralisation, l'affermissement des structures sociales et la progression du contrôle social sur le territoire. ${ }^{58}$ Après 1760 , rien de bien différent à cet égard, si ce n'est que les élites étaient mixtes et que les soldats et les immigrants étaient anglais. Comme auparavant, les paysans réglaient leurs litiges entre eux ou ils devaient le faire par l'entremise des seigneurs, des clercs, des officiers de milice et, maintenant, des juges de paix.

En l'occurrence, l'image d'un Etat autoritaire, tatillon en matière économique, dont le contrôle ne cesse de s'étendre et de peser sur tous, y compris la bourgeoisie, n'est pas étonnante. Pas plus d'ailleurs que celle d'une paysannerie soumise aux exigences des seigneurs, des curés et des responsables de la société militaire ne détonne. En tout cas, la vision de l'officier de milice, comme une sorte d'avant-garde de la 
démocratie populaire, ne s'applique pas. ${ }^{59}$ Dans cet univers contrôlé, il $n$ 'y eut place ni pour une religion populaire ni pour l'enracinement $d$ 'une solide volonté de résistance populaire. ${ }^{60} \mathrm{D}$ 'autant plus que de génération en génération à partir des pionniers, l'analphabétisme se diffusa dans la paysannerie jusqu'au siècle suivant, sans pour autant, amener les femmes à parité avec les hommes quant au niveau d'instruction. ${ }^{61}$

On ne saurait non plus comprendre la Nouvelle-France à moins de rappeler qu'elle était une société coloniale dominée par les métropolitains et, par eux aussi, rattachée à un empire construit et maintenu en fonction des intérêts de la métropole. Celle-ci non seulement monopolisait le trafic extérieur ${ }^{62}$ mais contrôlait dans toute la mesure possible les autres aspects de la vie coloniale. Dans la plupart des secteurs et des catégories sociales, en commençant par les gouverneurs et les intendants, on trouvait, surtout au sommet, une proportion variable de personnages dont le désir avoué ou non était de se recycler un jour en France. Ce furent eux qui quittèrent le pays en 1760, abandonnant ceux qui y avaient des intérêts permanents.

Dominés et exploités, les habitants n'en développèrent pas pour autant une conscience nationale. Mais le sentiment d'être né au pays, d'être français et catholique avait quand même nourri un sens d'identité canadienne, voire d'impatience entre coloniaux et métropolitains. Ceci dit, en 1750, presque personne n'envisageait l'avenir sans une métropole et la fidélité au roi.

S'il y eut une classe ébranlée par le changement de régime, ce fut la noblesse. D'un seul coup, elle se retrouva sans influence politique et sans rôle militaire. L'absence de substitut pour les troupes de la marine et l'abolition des milices en 1765 la laissèrent encore plus désemparée. Il ne lui resta bientôt, comme atout, que la conscience de son importance passée, et un domaine foncier menacé partiellement par la mise en vente des seigneuries des émigrants nobles. Mais, comme elle en racheta une partie et que le reste tomba surtout dans les mains d'anglophones du même rang qu'elle, l'avenir pouvait sembler moins sombre. Le caractère aristocratique de la société pouvait être sauvé.

Comme de raison, la noblesse se sentit tout aussi menacée par les mesures anti-féodales de la politique de 1763 et par la révolution américaine. Mais, dans sa lutte, elle put compter sur l'appui du clergé catholique, sur l'aide des gouverneurs et des défenseurs de l'ordre aristocratique. En 1774, elle recouvra pour un temps le sens de son pouvoir. Mais bientôt, elle commença à éprouver les effets du déclin économique, étant de plus en plus forcée de vendre des fiefs sans en racheter. Elle devint encore plus inquiète et plus militante lorsqu'après 1783 le mouvement en faveur des institutions parlementaires prit de l'ampleur. Une fois la réforme accomplie, elle aurait pu, au moins en 
partie, être rassurée par la place importante qu'elle occupait dans le nouvel ordre politique. Mais, lorsque la révolution s'en prit au roi et au clergé, sa peur des idées révolutionnaires se transforma en panique.

Le clergé éprouva certes des sentiments semblables à ceux de la noblesse au lendemain de 1760. Car, tout rapport avec Rome et la France était interdit. Bien plus, par la politique de 1763, la dîme, la Coutume de Paris et le régime seigneurial furent menacés d'extinction. Ajoutons à cela le déclin accéléré des ordres religieux mâles et le départ de prêtres qui forçèrent la colonie à dépendre du clergé séculier au moment où la noblesse en difficulté fournissait moins de candidats à la prêtrise qu'autrefois.

Mais, le clergé entreprit de reconstituer avec les Britanniques des rapports identiques à ceux qui avaient existé avec la France monarchique et absolutiste. La chose fut d'autant plus aisée que la vision gallicane des rapports entre l'Eglise et l'Etat était proche de l'anglicane. Ainsi, Briand devint évêque de Québec en 1765. Dès lors, grâce à l'absence d'immigration anglaise substantielle et à l'agitation dans les colonies atlantiques, les relations entre le clergé catholique et l'Etat colonial devinrent plus intimes, surtout après l'adoption de l'Acte de Québec. A son arrivée, trois décennies plus tard, le premier évêque anglican décrira l'Eglise catholique comme la seule établie et la sienne plutôt comme une secte tolérée.

Le clergé avait sans doute préservé l'essentiel de son domaine seigneurial et maintenu, en gros, avec les fidèles catholiques les rapports d'autrefois. Cependant, il était inquiet. Il le fut au sujet de la réaction paysanne au moment de la révolution américaine. Il le fut bien davantage à l'approche de la réforme de 1791 qu'il présenta plus tard comme l'origine de tous les maux. On ne peut s'étonner qu'il ait décrit la révolution française comme un cataclysme d'origine diabolique. ${ }^{63}$

La seconde moitié du XVIIIe siècle fut aussi celle de la montée de la bourgeoisie. Evidemment, les rapports de dépendance entre la métropole et la colonie existaient toujours et ils eurent leurs effets habituels sur le développement économique. La bourgeoisie coloniale, maintenant composée d'anglophones et de francophones, prit de l'ampleur grâce aux bénéfices tirés des secteurs traditionnels et, après 1783, grâce à l'accroissement de ses investissements dans la propriété seigneuriale. Avec le temps, cependant, les marchands canadiens furent distancés par les marchands britanniques, dont l'avance fut surtout remarquable dans les plus grosses entreprises. C'est dans cette foulée que le groupe des professionnels connut une expansion accélérée. Pour un temps après 1763 , ces deux groupes eurent l'air divisés sur nombre de points. Après 1783, toutefois, leurs revendications allèrent dans la même direction et, dès ce moment, il parût évident que les conflits déclenchés vers 1765, 
loin d'avoir eu un caractère nationaliste dominant, avaient d'abord opposé des classes. Car, pour la plupart des bourgeois engagés dans le mouvement pour les institutions parlementaires, l'objectif premier n'avait pas été la prise du pouvoir en vue d'opérer un changement social radical mais un partage de ce pouvoir à leur plus grand avantage économique. ${ }^{64}$

Il est vrai qu'il existait une rhétorique diffusée par la Gazette de Montréal, par des pamphlets et des chansons qui laissaient croire à l'existence d'un esprit révolutionnaire dans une fraction importante de la bourgeoisie. Les célébrations et les santés à l'occasion de la Révolution française et de la Constitution de 1791 donnèrent une certaine crédibilité à cette croyance. Mais tout cela semble venir de sources limitées et n'avoir agi que superficiellement sur l'opinion. A ce sujet, le livre de Claude Galarneau ${ }^{65}$ est certainement un meilleur guide que les écrits de Tousignant, Wallot et Greenwood. Quoi qu'il en soit, parmi ces bourgeois, le virage idéologique fut tout aussi brutal en 1793 qu'il le fut parmi les défenseurs de l'ordre aristocratique.

A vrai dire, comment pourrait-on arriver à une interprétation plausible de ces mouvements sans faire référence aux sursauts d'humeur populaire? Rappelons d'abord qu'à la conquête les Britanniques avaient désarmé les miliciens. En 1763, ils avaient voulu lever, avec assez de succès doit-on dire, un corps de volontaires pour aller combattre une insurrection indienne. Contrairement à ce qui se pratiquait auparavant, le gouvernement avait défrayé le coût de l'équipement et rémunéré la troupe. Mais, deux ans plus tard, les milices de la colonie furent abolies. Tout cela bouleversait les normes traditionnelles de l'exercice du devoir militaire. Une décennie plus tard, se produisit un conflit armé entre la Grande-Bretagne et ses colonies. Le gouvernement tenta alors, comme cela se pratiquait dans la métropole, de mobiliser, toujours moyennant rémunération, les paysans contre l'ennemi. Pendant ces années, les récoltes donnaient de gros surplus et des bruits persistants circulaient que les Britanniques voulaient les conscrire pour mieux leur infliger le sort des Acadiens. On comprend que les paysans aient été confus, tiraillés et agités; qu'ils aient finalement résisté à l'appel des hommes politiques et de leurs élites et opté en général pour une sorte de neutralité. Peu de choses en cela, surtout pas les dires de ceux qui furent déçus par leur conduite, qui permettent d'évoquer l'existence d'un véritable climat pré-révolutionnaire.

Il est encore plus difficile de connaître la réaction de ces milieux populaires face aux pressions de ceux qui oeuvraient pour ou contre dans le mouvement en faveur des institutions parlementaires. Car, chacun des adversaires tenta de les annexer à son plan. Il semble cependant qu'en général les paysans et les artisans ordinaires eurent de la 
peine à se former une opinion ferme. Il est certain, en tout cas, qu'ils ne furent pas insensibles aux mises en garde des nobles qui présentaient la chambre d'assemblée comme une machine à taxer.

A lire Murray Greenwood, on a l'impression que ces milieux populaires qui constituaient le gros de l'électorat, étaient déjà à cette date hostiles au régime seigneurial et très anti-anglais. Pourtant, plus de la moitié des députés élus en 1792 étaient des seigneurs nobles ou bourgeois. Dans ce même groupe de représentants du peuple, les Britanniques étaient également sur-représentés avec un tiers des députés venant de comtés urbains et ruraux. Lorsque l'argument ethnique fut présenté, ce fut sans succès en général. Fait intéressant à noter, cet équilibre ne fut guère modifié par les deux élections suivantes. ${ }^{66}$

N'empêche qu'après 1791, la province connut plusieurs poussées d'agitation populaire déclenchée à la suite de législations sur les milices et la voirie. A quelques occasions aussi, des censitaires s'opposèrent à leurs seigneurs sur des points similaires. Notons que ces mesures comportaient presque toujours, directement ou indirectement, des levées de taxes, soit de la part de l'Assemblée législative, de l'Etat lui-même ou des seigneurs. Qu'il s'agisse de service militaire ou de corvées, ces mesures aboutissaient à distraire, souvent à contretemps, une partie de la main-d'oeuvre des fermes. Spontanément, les dirigeants politiques, qui voyaient derrière ces mouvements de peuple la main des agents de la France révolutionnaire, réagirent vivement et le calme revint.

Mais, pour rendre compte de ces échecs, les historiens mentionnent à tout coup le manque de leadership. Pourtant, ils affirment avec la même conviction que, depuis 1774, il existait de véritables partis politiques aux tendances démocratiques et nationalistes. Dès la première session de 1792, ajoutent-ils, dans cette assemblée législative à $70 \%$ francophone, ces partis se polarisèrent selon la ligne ethnique et, dès lors, le parti canadien mit de l'avant une idéologie nationaliste et libérale. Si cela est vrai, comment expliquer qu'au lieu de contribuer à des législations réactionnaires affectant surtout l'ensemble de la population d'origine française, cette formation politique n'ait même pas tenté de mobiliser ces fièvres populaires à son profit? Pour rendre compte de ces contraditions, on parle d'absentéisme et de la pauvreté plus grande des députés francophones, sans se demander s'il n'y avait pas derrière ces gestes un ingrédient tactique. Il est clair qu'en résistant sans évocation idéologique à des mesures ponctuelles qui les heurtaient, les agitateurs populaires ne désiraient aucunement remettre en question le régime seigneurial et l'Etat féodal comme tels. N'était-ce pas plutôt la Chambre d'Assemblée qui était jusqu'à un certain point visée en tant que machine à taxer?

Il apparaît aussi que la bourgeoisie, francophone et anglophone, fut elle aussi happée par le mouvement de peur morbide qui regroupa les 
dirigeants politiques aussi bien que les élites contre la Révolution française et contre toute agitation populaire qui aurait pu servir à la diffusion de son message. Murray Greenwood propose l'idée de mentalité assiégée pour rendre compte du comportement des anglophones. Non seulement le mot est-il excessif pour un fait de conjoncture à propos d'une population dont une bonne proportion était bien enracinée dans le pays et diversifiée socialement. Mais, dans la mesure où les réactions émotives des élites canadiennes-françaises furent sans exception les mêmes que celles de leurs équivalents d'origine britannique, le vocable, s'il s'applique, doit l'être pour les deux. Il est évident que Greenwood ne saurait aller jusque-là.

$\mathrm{Au}$ fond, ce qui est le plus frappant en tout cela, c'est que la société québécoise, ou si on veut ses élites, a vécu pendant plus d'un siècle avec une seule version de son passé. Pourtant, c'est l'époque où, après 1850, un brassage démographique extraordinaire s'est produit: colonisation des régions périphériques, émigration massive vers les Etats-Unis et le reste du Canada, et urbanisation; enfin, mise en place d'une société industrielle. Beaucoup, comme si cette reconstruction du passé autour de l'idée de nation érigée sur la race, la langue et la religion aurait pu suffire à arrêter à elle seule la marche vers les inévitables. L'image repoussante des Révolutions américaine et française étant une des armes employées pour obtenir ce résultat.

Mais, dès le moment où se dessine la Révolution tranquille, ces révolutions furent récupérées pour d'autres objectifs classés sous le vocable modernisation. Dans les années 1970, ce fut pour un grand nombre d'intellectuels la récupération de la Révolution russe. Dès lors, les versions du passé, y compris le XVIIIe siècle, se multiplièrent, s'opposèrent et s'entremêlèrent pour une histoire sans fin, désormais ouverte, nous l'espérons, aux controverses et résistante à l'unanimité.

\section{FERNAND OUELLET}

York University

\section{Notes}

1 F. Ouellet, 'Historiographie canadienne et nationalisme,' dans les Mémoires de la Société Royale du Canada, 1975, 25-39.

2 F. Ouellet, 'La formation d'une société dans la vallée du Saint-Laurent. D'une société sans classes à une société de classes,' Canadian Historical Review (CHR), 1981, 402-451;'La tradition révolutionnaire au Canada. A propos de l'historiographie des Insurrestions de 1837-38 dans le Bas-Canada,' La revue de l'Université d'Ottawa (RUO), 1985, 91-125; 'Race et survivance nationale chez François-Xavier Garneau,' à paraître dans Etudes françaises; Serge Gagnon, Le 
Québec et ses historiens de 1840 à 1920. La Nouvelle-France de Garneau à Groulx (Québec: PUL, 1878) 288-324; P. Savard, 'François-Xavier Garneau,' dans L. Lapierre (éd.), Four $O^{\prime}$ Clock Lectures. French Canadian Thinkers of the Nineteenth and Twentieth Centuries (Montréal: McGill, 1966) 23-39.

3 Garneau, Histoire du Canada, 5e édition, (Paris: Alcan, 1920) vol. II, 662-66, 676, 679.

4 Histoire du Canada depuis la découverte jusqu'à nos jours (Québec: Aubin, Fréchette et Lowell, 1845-52) vol. I,23,25; Histoire du Canada, 5e éd., II, 346,717.

5 Ibid., 92. Ce commentaire est fait lorsque Garneau se réjouit que le roi de France n'ait pas introduit l'esclavage au Canada.

6 Ibid., I, 365.

7 Ibid., I, 34, 231, 234.

8 Ibid., I, 202, 208, 217; II, 447.

9 Ibid., I, 219.

10 Ibid., I, 475.

11 Ibid., I, L1, XLLIX, 339; II, 418, 427, 436.

12 Ibid., II, 348, 370, 384, 392.

13 Ibid., II, 439-443.

14 Ibid., 715-718.

15 F. Ouellet, Economy, Class, and Nation in Quebec. Interpretive Essays (Toronto: Copp Clark, 1991) 126, 242.

16 F. Ouellet, The Socialization of Quebec Historiography since 1960 (Toronto: York University, 1988) 7-9.

17 Lionel Groulx, Histoire du Canada français depuis la découverte (Montréal: AN, 1952) IV, 197.

18 F. Ouellet, Economy, Class, and Nation in Quebec..., 10-15; voir aussi Esther Delisle, Le Traitre et le Juif. Lionel Groulx, Le Devoir, et le délire du nationalisme d'extrême-droite dans la province de Québec, 1929-1939 (Outremont: L'Etincelle, 1992).

19 L. Groulx, Histoire du Canada..., I, 97,208.

20 Ibid., II,258; Notre Maître, le Passé, II, 261.

21 Histoire du Canada..., I, 70, 79, 109, 163, 187; II, 111, 161, 163, 165, 167, 172, 176, 204; Notre Maître, le Passé, II, 263, 272; L'Appel de la race (Montréal, AF, 1922), 281pp.

22 Histoire du Canada..., I, 204; II, 193.

23 Ibid., III, 76.

24 Ibid., III, 83-87.

25 Ibid., III, 9.

26 Ibid., III, 87.

27 Ibid., III, 138.

28 Ibid., III, 118-120.

29 Ibid., III, 100ss.

30 Guy Frégault, La civilisation de la Nouvelle-France (Montréal, Editions Pascal, 1944), 42, 68, 83, 118, 134, 172, 182-186, 220, 222, 239, 276. 
31 F. Ouellet, 'La formation d'une société dans la vallée du Saint-Laurent...,' CHR, 1981, 423-26.

32 Maurice Séguin, L'idée d'indépendance au Québec: genèse et historique (Trois-Rivières: Boréal Express,1967).

33 Michel Brunet, Canadiens et Canadians. Etudes sur l'histoire et la pensée des deux Canadas (Montréal: Fides, 1954).

34 M. Brunet, Les Canadiens après la Conquête, 1759-1775 (Montréal: Fides, 1969).

35 Pierre Tousignant, 'Les aspirations libérales des réformistes canadiens-français et la séduction du modèle constitutionnel britannique, de 1789 à 1792,' dans Sylvain Simard, La Révolution française au Canada français (Ottawa: PUO, 1991) 229-239.

36 Jean-Pierre Wallot, Un Québec qui bougeait. Trame socio-politique au tournant du XIXe siècle (Montréal: Boréal Express,1973) 253-319.

37 Murray Greenwood, Legacies of Fear. Law and Politics in Quebec in the era of the French revolution (Toronto: UTP, 1993) 35-171.

38 Donald Creighton, The Dominion of the North (Toronto: UTP, 1957) 77, 85, 117s., 120, 126, 128; The Empire of the St. Lawrence (Toronto: Macmillan, 1956) V, 2, 23, 35, 117,110 .

39 Denis Monière, Le développement des idéologies au Québec (Montréal: Québec/Amérique, 1977); Gilles Bourque, Classes sociales et Question nationale au Québec (Montréal: Parti-Pris, 1970); F. Ouellet, Economy, Class, and Nation in Quebec... 28s., 236-39, 251-262.

40 F. Ouellet, 'L'historiographie québécoise des années 1980' dans Luigi Bruti Leberati e Fabricio Ghilardi (éd.), Canada Ieri E Oggi 3 (Torre Canne: Schena, 1992) 51-79.

41 F. Ouellet, 'Accroissement de la population catholique québécoise avant 1850: aperçus historiographiques et quantitatifs,' L'Actualité économique, 1983, 402-422; Jacques Henripin et Yves Péron, 'La transition démographique de la Province de Québec,' dans H. Charbonneau (éd.), La population du Québec. Etudes rétrospectives (Montréal: Boréal Express, 1973) 23-44.

42 Hubert Charbonneau et al., Naissance d'une population. Les Français établis au Canada au XVIIe siècle (Montréal: PUM, 1987).

43 Marcel Fournier, Les Européens (hors France) au Canada des origines à 1765 (Montréal: Editions du Fleuve, 1989).

44 F. Ouellet, Economy, Class, and Nation in Quebec..., 126; Lorraine Gadoury, Yves Landry et Hubert Charbonneau, 'Démographie différentielle en Nouvelle-France: villes et campagnes,' RHAF, 1985, 357-378. Les auteurs montrent que les villes eurent déjà et dès le début un comportement démographique nettement différent de celui des campagnes.

45 F.Ouellet, Ibid., 129; Sylvie Dépatie, 'La transmission du patrimoine dans les terroirs en expansion: un exemple canadien au XVIIIe siècle, RHAF, 1990, 171-198; Yves Landry et Réal Bates, 'Population et reproduction sociale à l'lle d'Orléans aux XVIIe et XVIIIe siècles,' RHAF, 1992, 403-414; Louis Lavallée, 'La vie et la pratique d'un notaire rural sous le Régime français: le cas de Guillaume Barette, notaire à Laprairie entre 1709-1744,' RHAF, 1994, 499-520. 
46 André Lachance, Crimes et Criminels en Nouvelle-France (Montréal: Boréal Express, 1984) 76-79; Marie-Aimée Cliche, 'Filles-mères, familles et société sous le Régime français,' Histoire sociale-Social History (HS-SH), 1988, 39-70.

47 Louise Dechêne, Habitants et marchands de Montréal au XVIIe siècle (Montréal, 1974). L'auteure exagère l'étendue du cloisonnement intersectoriel et sous-estime grossièrement le volume de la main-d'oeuvre engagée dans la traite ainsi que son origine rurale.

48 F. Ouellet, Economy, Class, and Nation in Quebec..., 121-229.

49 L. Dechêne, Le partage des subsistances au Canada sous le régime français (Montréal: Boréal, 1994) 16, 21, 203. Douze années d'exportations de blé de 1721 à 1760, soit environ $16.5 \%$ de la récolte, dit-elle, et douze années d'importations de farine de 1738 à 1760 .

50 F. Ouellet, Economy, Class and Nation in Quebec..., 72-77.

51 Daniel Francis et Toby Morantz, La traite des fourrures dans l'est de la Baie James, 1600-1870 (Montréal: PUQ, 1984; Silvia Van Kirk, 'Many Tender Ties.' Women in Fur-Trade Society, 1670-1870 (Winnipeg: Watson and Dwyer, 1980); Jean-François Brière, La pêche française en Amérique du Nord au XVIIIe siècle (Montréal, Fides, 1990); F. Ouellet, 'Développement et sous-développement en milieu colonial pré-industriel,' Acadiensis, 1992, 146-168. Discussion de la littérature centrée sur le rôle de la main-d'oeuvre saisonnière dans les économies où pêche, pelleteries et forêt étaient en interaction avec le monde agricole.

52 F. Ouellet, 'Officiers de milice et structure sociale au Québec, 1660-1815,' dans HS-SH, 1979, 37-65.

53 Pour une analyse de la nature et du rôle de la noblesse (milices, armée régulière, haut clergé, propriété foncière, traite et politique), voir F. Ouellet, Economy, Class, and Nation in Quebec..., 6-87; pour une étude plus restrictive, voir Lorraine Gadoury, La noblesse en Nouvelle-France (Montréal: HMH, 1991).

54 Jean-Claude Dubé, Les Intendants de la Nouvelle-France (Montréal: Fides, 1984).

55 Paul Lemieux, ' Le clergé catholique de la vallée du Saint-Laurent, 1756-1810: évolution numérique, origine géographique et origine sociale,' thèse de maîtrise, Université d'Ottawa, 1986, 157pp. Les séries quantitatives les plus importantes remontent au début du XVIIe siècle; Marguerite Jean, Evolution des communautés de femmes au Canada de 1639 à nos jours (Montréal: Fides, 1977) 9-62; Serge Gagnon et Louise Lebel-Gagnon, 'Le milieu d'origine du clergé québécois, 1775-1840: mythes et réalités,' RHAF, 1983, 373-397.

56 L. Dechêne, Le partage des subsistances au Canada sous le Régime français, 10.

57 Sylvie Dépatie, Mario Lalancette, Christian Dessureault, Contributions à l'étude du régime seigneurial canadien (Montréal: $\mathrm{HMH}, 1987$ ); Allan Greer, Peasant, Lord and Merchant. Rural Society in Three Parishes, 1740-1840 (Toronto: UTP, 1985); F.

Ouellet, 'Libéré ou exploité? Le paysan québécois d'avant 1850,' HS-SH, 1980, 339-368.

58 John Alexander Dickinson, Justices et justiciables. La procédure civile à la prévôté de Québec, 1667-1759 (Québec: PUL, 1982) 142-146, 162-164; A. Lachance, Crimes et Criminels en Nouvelle-France, 77-83; André Cellard, Histoire de la folie au Québec de 1600 à 1850 (Montréal: Boréal, 1988). 
59 F. Ouellet, 'The British Army of Occupation in the St. Lawrence Valley, 1760-1774,' in R.A. Prete and A.H. Ion (ed.), Armies of Occupation (Waterloo: WLUP, 1984) 17-54.

60 M.-Aimée Cliche, Les pratiques de dévotion en Nouvelle-France. Comportements populaires et développement ecclésial (Québec: PUL, 1988).

61 Michel Verrette, 'L'alphabétisation de la population de la ville de Québec de 1750 à 1849,' RHAF, 1985, 68, 72; H. Charbonneau et al., Naissance d'une population..., 160; A. Greer, 'L'alphabétisation et son histoire au Québec,' dans Yvan Lamonde (éd.), L'Imprimé au Québec. Aspects historiques (Québec: IGRE, 1983) 41-45.

62 Jacques Mathieu, Le commerce entre la Nouvelle-France et les Antilles au XVIIIe siècle (Montréal: Fides, 1981); F. Ouellet, Economy, Class, and Nation in Quebec..., 210-233.

63 F. Ouellet, Le Bas-Canada, 1791-1840. Changements structuraux et crise (Ottawa: PUO, 1976) 13-80.

64 F. Ouellet, Ibid., 30-35.

65 Claude Galarneau, La France devant l'opinion canadienne (1760-1815) (Québec: PUL, 1970) $55-105,155-259$.

66 F. Ouellet, Le Bas-Canada..., 41-45, 73-80. 